\title{
Importance of right ventricular outflow tract angiography in distinguishing critical pulmonary stenosis from pulmonary atresia
}

\author{
Kevin P Walsh, Jassim M Abdulhamed, Andrew J P Tometzki
}

\begin{abstract}
Objective-To investigate the spectrum of pulmonary atresia and critical pulmonary stenosis using right ventricular outflow tract angiography and explore its implications for catheter interventional treatment.
\end{abstract}

Design-Prospective clinical study. Setting-Two paediatric cardiology centres.

Subjects-11 neonates or infants (aged 1 day to 8 months; weighing 2.3 to $7.8 \mathrm{~kg}$ ) with pulmonary atresia or where the differentiation of pulmonary atresia from critical pulmonary stenosis was unclear on either echocardiography or angiography.

Methods-Right ventricular outflow tract angiography was performed on all patients to distinguish pulmonary atresia from critical pulmonary stenosis before opening the right ventricular outflow tract.

Results-Right ventricular outflow tract angiography showed that three of seven patients diagnosed as pulmonary atresia by echocardiography had pin hole jets across the pulmonary valve; another had a probe patent valve that appeared imperforate on both echocardiography and right ventricular outflow tract angiography. Three of the four patients diagnosed by echocardiography as critical pulmonary stenosis were found on right ventricular outflow tract angiography to have pulmonary atresia. The remaining patient had such a tiny orifice that a second orifice had to be created with a radiofrequency catheter. The right ventricular outflow tract was opened successfully in 10 of the 11 patients, six of whom required application of radiofrequency energy. The right ventricular to aortic systolic pressure ratio fell from $1.4(0.9$ to $1.9)$ to $0.6(0.2$ to 1.1$)(P<0.05)$. All 11 patients were alive and well with transcutaneous oxygen saturations ranging from $84 \%$ to $95 \%$ at a median follow up duration of nine months.

Conclusions-Critical pulmonary stenosis and pulmonary atresia cannot always be accurately distinguished by echocardiography. Right ventricular outflow tract angiography helps to distinguish the two groups. In most cases the right ventricular outflow tract can be opened without mortality and with short to medium term survival.
(Heart 1997;77:456-460)

Keywords: pulmonary atresia; critical pulmonary stenosis; radiofrequency perforation; angiography

Pulmonary atresia with intact ventricular septum remains a difficult condition to treat. Prognosis and management depends on the degree of ventricular hypoplasia and number of parts of the ventricular cavity remaining, and in particular whether the infundibulum is present. ${ }^{1}$ ${ }^{2}$ Right ventricular to coronary artery sinusoids with coronary artery stenoses may produce a right ventricular dependent myocardial blood supply, preventing right ventricular decompression. ${ }^{3}$ Surgical strategies consist of systemic to pulmonary shunt, with or without pulmonary valvotomy or right ventricular outflow patch, depending on the presence of an infundibulum. ${ }^{4}$ During closed surgical pulmonary valvotomy surgeons often remark that there is a tiny jet of blood coming through a pin hole orifice. The distinction between critical pulmonary stenosis and pulmonary atresia may not be completely clear cut even after angiographic studies. Recently laser and radiofrequency assisted perforation of membranous atretic pulmonary valves has been described, with a mortality of between $25 \%$ and $50 \% .{ }^{5-8}$ These results may reflect patient selection and learning curve effects. Improved results with these techniques requires clear delineation of the atretic pulmonary valve anatomy. We looked at the spectrum of pulmonary atresia and critical pulmonary stenosis and its implications for catheter interventional treatment.

\section{Methods}

Twenty one patients presented with pulmonary atresia or critical pulmonary stenosis and intact ventricular septum between October 1993 and May 1995 to our two hospitals. Cross sectional echocardiography with Doppler and colour flow mapping was performed in all patients using a Hewlett Packard Sonos 500 with a $5.0 \mathrm{MHz}$ ultrasound probe. Images were obtained to show right ventricle to pulmonary artery continuity, valve diameter and thickness, arterial duct patency, and the degree of right ventricular hypoplasia. Patients without an infundibular portion of a hypoplastic right ventricle were not included in this study. Whether the valve was atretic or patent was assessed with pulsed and continuous wave Doppler and colour flow mapping.

Ten patients had clear cut critical pulmonary
Royal Liverpool Children's Hospital, Alder Hey, Eaton Road, Liverpool L12 2AP,

Accepted for publication 12 February 1996 
stenosis shown by echocardiography and confirmed by angiography and are excluded from further consideration. Our study group consists of 11 patients with pulmonary atresia or in whom the differentiation of pulmonary atresia from critical pulmonary stenosis was unclear on either echocardiography or angiography. These 11 patients were aged between 1 day and 8 months (median 58 days) and weighed 2.3 to $7.8 \mathrm{~kg}$ (median $3.5 \mathrm{~kg}$ ). Eight of the 11 patients were receiving prostaglandin $\mathrm{E}_{1}$ or $\mathrm{E}_{2}$ infusions.

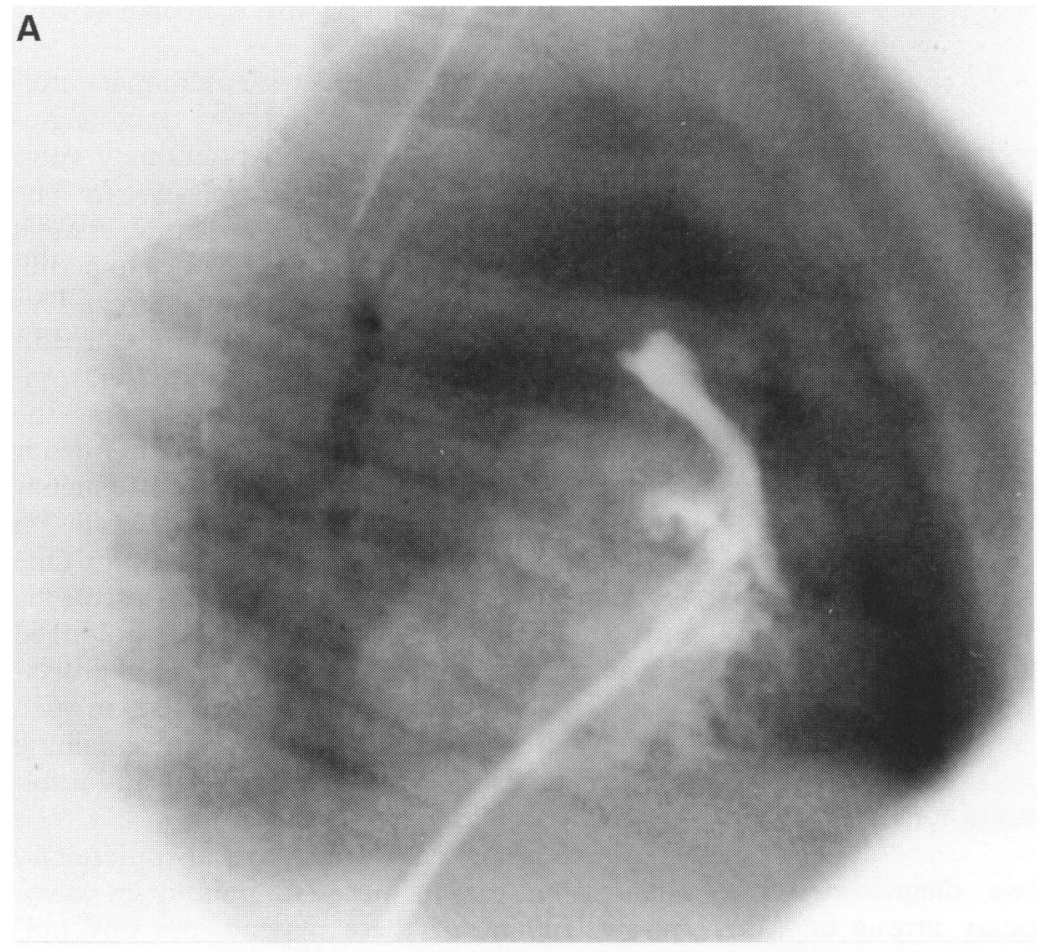

B

Figure 1 (A) Right ventricular body angiogram (lateral projection) fails to show an anterograde jet. (B) Right ventricular outflow tract angiogram shows a small anterograde jet and the patient has critical pulmonary stenosis rather than pulmonary atresia. There is also a catheter placed retrogradely in the pulmonary artery through a modified BlalockTaussig shunt.
Two patients aged 2 and 8 months had persistently patent arterial ducts without prostaglandin infusion. One patient aged 2 months had previously undergone a right modified Blalock-Taussig shunt for presumed pulmonary atresia. Three patients had previously undergone cardiac catheterisation and right ventricular body angiograms.

\section{TECHNIQUE}

Cardiac catheterisation and angiography was performed under general anaesthesia in all patients before dilatation of the pulmonary valve. Angiographic patency of the pulmonary valve was assessed from right ventricular outflow tract injections. We used a modification of the technique described by Rosenthal et $a l^{5}$ to perforate the atretic pulmonary valve. A right coronary Judkins catheter $(4,4 \cdot 5,5$, or $6 \mathrm{~F})$ was placed in the right ventricular outflow tract immediately beneath the centre of the pulmonary valve. Injection of small volumes of dye by hand in the right ventricular outflow tract was used to aid positioning. The double catheter technique with simultaneous right ventricular outflow tract and main pulmonary artery angiograms was used in five of the 11 patients. If the valve was patent it was crossed with a 0.014 inch (Microvena, Ultraselect) or 0.018 inch (ACS) guidewire. If the valve was atretic it was perforated with a $2 \mathrm{~F}$ radiofrequency catheter (Cerablate PA 120, Osypka). This catheter has a $3 \mathrm{~cm}$ flexible end with a $3 \mathrm{~mm}$ metal tip which is heated by applying 3 to $25 \mathrm{~W} / \mathrm{s}$ of $550 \mathrm{kHz}$ radiofrequency energy in unipolar mode, with a back plate serving as the indifferent electrode. Frequent rises in impedance required repeated applications of energy before the catheter could be advanced anterogradely into the main pulmonary artery. Once the atretic valve has been perforated, the radiofrequency catheter is removed and the newly created pinhole orifice is crossed with a high torque floppy guidewire, as for critical pulmonary stenosis. If possible this wire is passed into the distal abdominal aorta through the patent arterial duct in order to place the rigid part of the wire across the pulmonary valve to aid passage of the valvoplasty balloon. A low profile 3.5 or $4 \mathrm{~mm}$ diameter coronary angioplasty balloon (Medtronic Gold or USCI ULP) is then passed over this wire and inflated to enlarge the pulmonary valve orifice enough to allow the passage of a diagnostic catheter. This catheter is used to pass a larger diameter exchange guidewire to allow introduction of larger diameter valvoplasty balloon catheters. In six patients, the end of the 0.014 inch or 0.018 inch floppy guidewire was snared with a $5 \mathrm{~mm}$ diameter Amplatz goose neck snare (Microvena) and pulled out of the arterial sheath to create an arteriovenous guidewire loop. This arteriovenous guidewire loop facilitates secure passage of further dilatation balloons. The final size of the valvoplasty balloons was chosen to exceed the measured pulmonary valve annulus by at least $25 \%$.

STATISTICAL ANALYSIS

Results are given as mean (SEM) or median 


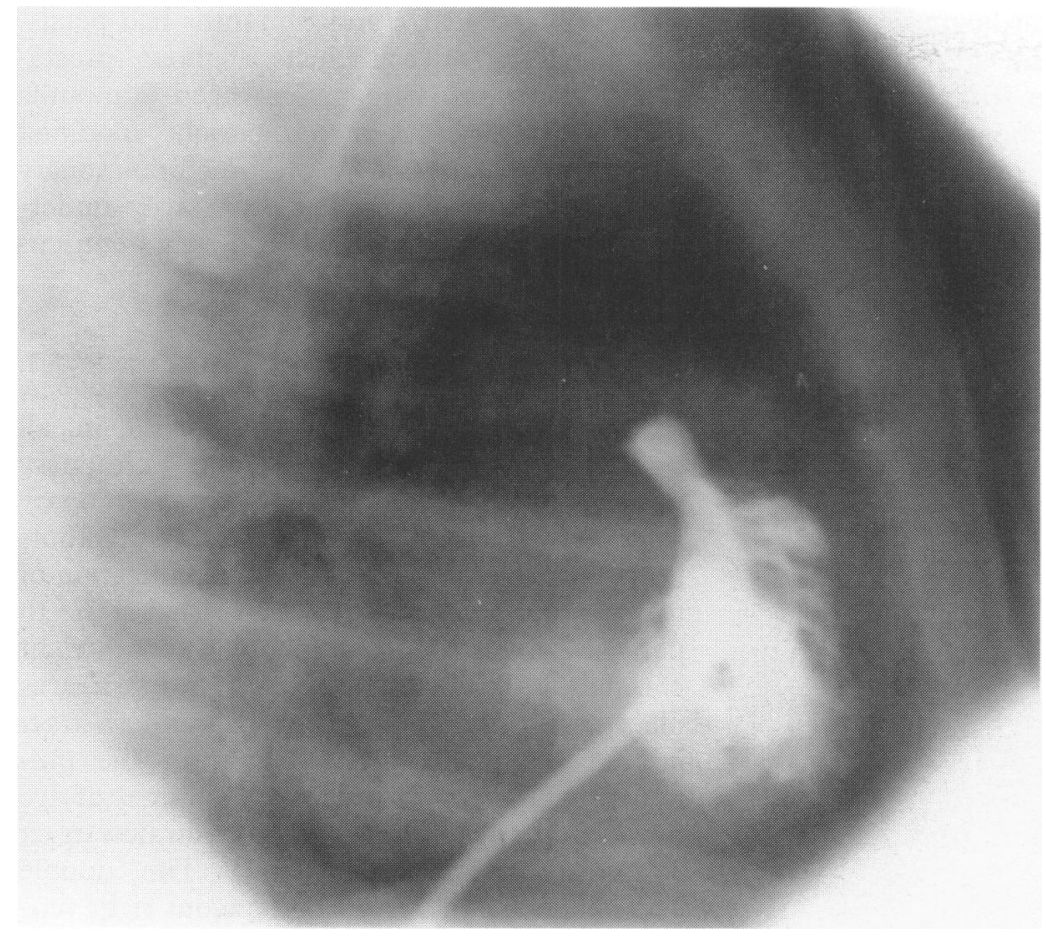

Figure 2 Right ventricular outflow tract angiogram shows no anterograde jet. The valve was crossed with the radiofrequency catheter without application of radiofrequency energy. This patient can be considered to have a probe patent atretic valve.

(range). A Student's $t$ test for paired data was used when appropriate. $P$ values less than 0.05 are considered to be statistically significant.

\section{Results}

CORRELATION OF ECHOCARDIOGRAPHIC AND ANGIOGRAPHIC STUDIES

With echocardiography we diagnosed seven patients as having pulmonary atresia and the remaining four as having critical pulmonary stenosis. Right ventricular outflow tract angiography was used as the gold standard and showed that three of the seven patients diagnosed as having pulmonary atresia had pin hole jets across the valve and hence had critical pulmonary stenosis on this basis. One of these three patients had previously undergone cardiac catheterisation with a right ventricular body angiogram which was interpreted as showing pulmonary atresia (fig 1). One patient was shown on both echocardiography and right ventricular outflow tract angiography to have pulmonary atresia but in fact had a probe patent valve, that is, the valve was "inadvertently" crossed by the radiofrequency catheter before application of radiofrequency energy (fig 2).

Three of the four patients diagnosed by echocardiography as having critical pulmonary stenosis were found on right ventricular outflow tract angiography to have pulmonary atresia. Two of these patients had previously undergone cardiac catheterisation with right ventricular body injections which had been interpreted as showing critical pulmonary stenosis, but in both cases a guidewire had failed to cross the pulmonary valve during the previous procedure. Another of these four patients with an echocardiographic diagnosis of critical pulmonary stenosis had such a small pulmonary valve orifice that an 0.018 inch guidewire could not be passed across it and a second larger orifice was created with a radiofrequency catheter to permit passage of a guidewire (fig 3).

\section{PROCEDURAL RESULTS}

Valvoplasty was accomplished in 10 of the 11 patients, with a maximum balloon diameter ranging from 8 to $10 \mathrm{~mm}$. In these patients the right ventricular pressure fell from 113 (SEM 9) $\mathrm{mm} \mathrm{Hg}$ to 51 (5) $\mathrm{mm} \mathrm{Hg}(\mathrm{P}<0.05)$ and the right ventricular to aortic systolic pressure ratio fell from $1.4(0.9$ to 1.9$)$ to $0.6(0.2$ to 1.1$)$ $(\mathbf{P}<0.05)$. Radiographic screening time ranged from 16 to $92 \cdot 2$ minutes, with a median of 55.3 minutes.

Six of the seven patients with pulmonary atresia on right ventricular outflow tract angiography underwent successful pulmonary valve perforation followed by valvoplasty. In one patient we could not obtain a central enough catheter position below the atretic valve to permit safe application of radiofrequency energy. This patient was the sole procedural failure in this series and he later underwent a successful surgical valvotomy. In one of the six patients the valve was crossed by the radiofrequency catheter without applying radiofrequency energy, although it appeared imperforate on right ventricular outflow tract angiography. One patient underwent balloon angioplasty of the arterial duct to avoid the need for prolonged prostaglandin administration. Prolonged prostaglandin administration was required in two patients (for 15 and 24 days). The remaining three patients did not require prostaglandin following valvoplasty.

All four patients with critical pulmonary stenosis underwent successful pulmonary valvoplasty. In one of these patients the tiny pulmonary valve orifice could not be crossed with a 0.018 inch high torque floppy guidewire (a smaller diameter guidewire was not tried) and a second orifice was therefore created with a radiofrequency catheter. Two of the four patients with critical pulmonary stenosis appeared to have extreme right ventricular hypoplasia which would have required either very prolonged prostaglandin administration or a surgical shunt, and they therefore underwent endovascular stent implantation in the arterial duct (P-S 20, Johnson and Johnson Interventional Systems). One patient had already had a surgical shunt for presumed pulmonary atresia. The patient in whom a second orifice was created with a radiofrequency catheter to permit valvoplasty received a prostaglandin infusion for 10 days.

\section{COMPLICATIONS}

One patient weighing $2 \cdot 3 \mathrm{~kg}$ developed inferior vena caval thrombosis resulting in abdominal distension and renal failure. She had undergone cardiac catheterisation the previous day in an unsuccessful attempt to treat presumed critical pulmonary stenosis. The following day she underwent successful radiofrequency perforation of pulmonary atresia. A triple lumen catheter was placed in the same femoral vein used for the valvoplasty at the end of the procedure. She required peritoneal dialysis and the 


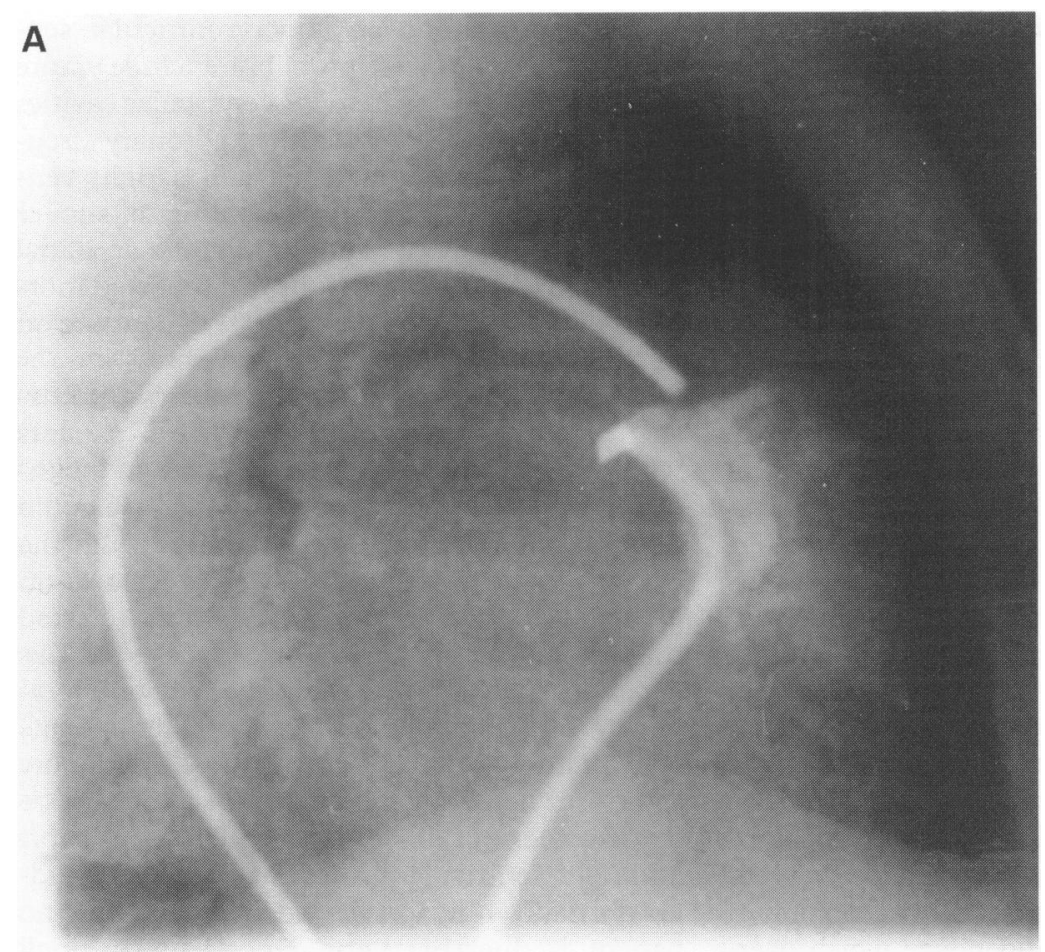

B same day as the procedure and four patients required prolonged intubation and ventilation of $8,8,15$, and 21 days.

\section{FOLLOW UP}

All patients were alive and well at a median follow up duration of nine months (range three to 21 months). All were clinically pink in colour with saturations measured by transcutaneous oximeters ranging from $84 \%$ to $95 \%$ (median $91 \%$ ). Follow up echocardiography showed a patent right ventricular outflow tract in all patients, with a peak pulmonary valve Doppler velocity ranging from 1.3 to $3.5 \mathrm{~m} / \mathrm{s}$ (median $2.1 \mathrm{~m} / \mathrm{s}$ ). The right ventricle appeared to have grown in all patients, although formal measurements of right ventricular volume have not been made. In one patient in whom a ductal stent was implanted the stent had occluded by three months without clinical consequence. Follow up cardiac catheterisation has been performed in three patients. In the patient who had a surgical shunt and balloon atrial septostomy for presumed pulmonary atresia followed later by balloon valvoplasty for critical pulmonary stenosis, there was no gradient across the right ventricular outflow tract, and the aortic saturation was $92 \%$ increasing to $96 \%$ when the shunt and atrial septal defect were temporarily occluded. She will require shunt and atrial septal defect occlusion in the future. In two other patients (cases 7 and 9) repeat valvoplasty was performed 11 and nine months later to relieve residual gradients of 60 and $30 \mathrm{~mm} \mathrm{Hg}$, respectively.

\section{Discussion}

RIGHT VENTRICULAR OUTFLOW TRACT ANGIOGRAPHY VERSUS ECHOCARDIOGRAPHY

This group of selected patients illustrates the spectrum of pulmonary atresia and critical pulmonary stenosis. The similarity of pulmonary atresia to critical pulmonary stenosis is shown by our finding of a probe patent although angiographically imperforate atretic valve in one patient and an angiographically patent valve that could not be probed in another. With such tiny stenotic orifices it is readily apparent that colour flow mapping of the pulmonary valve area in the presence of considerable ductal flow into the main pulmonary artery could easily miss a tiny anterograde jet through the valve. In our series three out of seven patients thought to have pulmonary atresia on the basis of echocardiography were found to have critical pulmonary stenosis, and three of four patients thought to have critical pulmonary stenosis were found to have pulmonary atresia. However, if we add the 10 patients who had critical pulmonary stenosis on both echocardiography and right ventricular outflow tract angiography (whom we excluded earlier from detailed consideration), then 11 of 14 patients with critical pulmonary stenosis and four of seven patients with pulmonary atresia were correctly predicted by echocardiography. Previous studies have suggested that echocardiographic assessment of neonates with pulmonary atresia and intact ventricular septum correlates well with angiography and that neonates can undergo surgical intervention without cardiac dilatation. She had torrential ductal flow and she was therefore kept ventilated for eight days to control the systemic to pulmonary run off Her duct and aneurysm have since closed spontaneously. Six patients were extubated on the 
catheterisation..$^{9}$ This study did not employ right ventricular outflow tract angiography routinely and may well have labelled some cases of critical pulmonary stenosis as pulmonary atresia.

\section{RIGHT VENTRICULAR BODY $v$ OUTFLOW TRACT ANGIOGRAPHY}

We did not perform right ventricular body angiography in all patients because of the need to give multiple contrast injections to obtain optimal radiofrequency catheter position beneath the pulmonary valve. Therefore we do not have a prospective comparison between right ventricular body angiography and right ventricular outflow tract angiography in all patients. However, right ventricular body angiography was insufficient to discriminate all patients with critical pulmonary stenosis from those with pulmonary atresia, and vice versa. The reasons for the failure of the right ventricular body angiogram to show the tiny anterograde jets are the lack of proximity of the injecting catheter to the valve and the associated moderate tricuspid regurgitation due to the suprasystemic right ventricular pressure. This results in opacification of the right atrium and its appendage which overlies the right ventricular outflow tract. We identified three patients where previous catheter studies with right ventricular body angiograms had given an incorrect diagnosis. One was presumed to have pulmonary atresia, although the true diagnosis was critical pulmonary stenosis, while two were presumed to have critical pulmonary stenosis, though the true diagnosis was pulmonary atresia. It is possible that the valve could have become imperforate in the interval between studies in one of the latter two patients. Patient management was significantly altered by the right ventricular outflow tract angiogram, with the former patient undergoing conventional wire passage and balloon dilatation of the pulmonary valve and the latter two requiring radiofrequency catheter perforation of the pulmonary valve before balloon dilatation. However, right ventricular outflow tract angiography cannot be considered to be definitive, as shown by our patient with an angiographically imperforate but probe patent valve. It is possible that digital subtraction angiography might have shown a jet. However, it is more likely that the combination of a stable guiding catheter position immediately beneath the thinnest part of the valve and a solid tipped radiofrequency wire allowed the atretic valve to be crossed without radiofrequency energy. It may well be worth probing the atretic pulmonary valve with a guidewire before radiofrequency perforation. For the patient with an angiographically patent tiny orifice in whom we created a second orifice, probing the valve with a smaller diameter guidewire, for example 0.010 inch, might also have succeeded.

SAFETY AND EFFICACY OF OPENING THE RIGHT VENTRICULAR OUTFLOW TRACT BY CATHETER INTERVENTION

Our study did not attempt to answer the question of whether catheter interventional management is better than or an acceptable alternative to surgical management in patients with pul- monary atresia and an intact ventricular septum. Surgical series probably include more patients with unipartite right ventricular cavities and right ventricular dependent coronary circulations. ${ }^{14}$ However, patients with a right ventricular outflow tract, representing a similar patient group to ours and undergoing an initial closed surgical valvotomy, have a reported mortality of between $12.5 \%$ and $50 \%$ depending on the size and era of the reported series. ${ }^{110}$

We have shown that radiofrequency perforation could be performed in our seven patients with no false passages or perforations and without mortality. In one patient in whom we could not achieve a satisfactory position beneath the centre of the atretic valve we desisted from applying radiofrequency energy and referred the patient for surgery. On the basis of this experience we consider radiofrequency perforation to be a safe method of converting patients with pulmonary atresia into critical pulmonary stenosis where valvoplasty is considered to be the treatment of choice. Our follow up is short and right ventricular growth has not been studied formally but appears adequate in that no patient is duct or shunt dependent. A small degree of right to left shunting continues through the atrial septum, and no patient has yet had the two circulations completely separated. We would hope that the atrial communications would either close spontaneously or be suitable for device or surgical closure.

\section{CONCLUSIONS}

Our study shows that critical pulmonary stenosis and pulmonary atresia cannot always be accurately distinguished by echocardiography or even right ventricular body angiography. Right ventricular outflow tract angiography helps to distinguish the two groups. In most cases the right ventricular outflow tract can be opened with good medium term results.

1 Bull C, Kostelka M, Sorensen K, DeLeval M. Outcome measures for the neonatal management of pulmonary atreSurg 1994;107:359-66

2 Giglia TM, Jenkins KJ, Matitiau A, Mandell VS, Sanders $\mathrm{SP}$, Mayer JE, et al. Influence of right heart size on outcome in pulmonary atresia with intact ventricular septum. Circulation 1993;88:2248-56.

3 Gentles TL, Colan SD, Giglia TM, Mandell VS, Mayer JE Sanders SP. Right ventricular decompression and left ventricular function in pulmonary atresia with intact ventricutricular function in pulmonary atresia with intact ventricular septum. The influence of less
anomalies. Circulation 1993;88:183-8.

4 Hanley FL, Sade RM, Blackstone EH, Kirklin JW, Freedom $\mathrm{RM}$, Nanda NC, et al. Outcomes in neonatal pulmonary atresia with intact ventricular septum: a multiinstitutiona study. F Thorac Cardiovasc Surg 1993;105:406-27.

5 Rosenthal E, Qureshi SA, Chen-Chan K, Martin RP Skehan DJ, Jordan SC, et al. Radiofrequency-assisted balloon dilatation in patients with pulmonary valve atresia and intact ventricular septum. Br Heart $\mathcal{F}$ 1993;69:347-51.

6 Qureshi SA, Rosenthal E, Tynan M, Anjos R, Baker EJ Transcatheter laser-assisted balloon pulmonary valve dilation in pulmonic valve atresia. Am $\mathcal{F}$ Cardiol 1991;67 428-31.

7 Parsons JM, Rees MR, Gibbs JL. Percutaneous laser valvotomy with balloon dilatation of the pulmonary valve as primary treatment for pulmonary atresia. Br Heart $f 1991$ 66:36-8.

8 Rosenthal E, Qureshi SA, Kakadekar AP, Anjos R, Baker EJ, Tynan $M$. Technique of percutaneous laser-assisted valve dilatation for valvar atresia in congenital heart disease. $\mathrm{Br}$ Heart ₹ 1993;69:556-62

9 Leung MP, Mok CK, Hui PW. Echocardiographic assessment of neonates with pulmonary atresia and intact

10 Joshi SV, Brawn WJ, Mee RBB. Pulmonary atresia with intact ventricular septum. $\mathcal{f}$ Thorac Cardiovasc Surg 1986 ; 91:192-9. 